\title{
VERTICAL LORENTZ FORCE AND CROSS-FIELD CURRENTS IN THE PHOTOSPHERIC MAGNETIC FIELDS OF SOLAR ACTIVE REGIONS
}

\author{
Manolis K. Georgoulis and Barry J. LaBonte \\ Applied Physics Laboratory, Johns Hopkins University, 11100 Johns Hopkins Road, Laurel, MD 20723 \\ Received 2003 November 5; accepted 2004 July 19
}

\begin{abstract}
We demonstrate that the vertical Lorentz force and a corresponding lower limit of the cross-field electric current density can be calculated from vector magnetograms of solar active regions obtained at a single height in the solar atmosphere, provided that the vertical gradient of the magnetic field strength is known at this height. We use a predicted vertical magnetic field gradient derived from a previous analysis. By testing various force-free solutions, we find that the numerical accuracy of our method is satisfactory. Applying the method to active region photospheric vector magnetograms, we find vertical Lorentz forces ranging from several hundredths to a few tenths of the typical photospheric gravitational force, and typical cross-field current densities up to several times $10 \mathrm{~mA} \mathrm{~m}^{-2}$. The typical vertical current density is found to be $2-3$ times smaller, on the order of $10-15 \mathrm{~mA} \mathrm{~m}{ }^{-2}$. These differences are above the associated uncertainties. The values of the cross-field currents decrease in an averaged vector magnetogram, but the ratio of the cross-field to the vertical current density increases, also above the uncertainties. We conclude that the photospheric active region magnetic fields are not force-free, contrary to the conjectures of some recent studies.
\end{abstract}

Subject headings: Sun: atmosphere — Sun: magnetic fields — Sun: photosphere

\section{INTRODUCTION}

The components of the magnetic field vector in solar active regions (ARs) are routinely measured at only one height in the solar atmosphere, namely, on the photosphere. A few attempts have focused on obtaining chromospheric vector magnetograms (Metcalf et al. 1995; Lin et al. 1998; Leka \& Metcalf 2003), while coronal magnetography is still in very preliminary stages (e.g., Brosius et al. 1997; Lin et al. 2000). As a result, magnetic extrapolation techniques have been developed with an aim to calculate, rather than measure, the magnetic field vector above the solar photosphere. Extrapolating the observed magnetic field is a boundary value problem, with the observed vertical field component serving as the boundary condition. Most extrapolations rely on the force-free approximation, solving either the linear (e.g., Alissandrakis 1981; Gary 1989) or the nonlinear (e.g., Amari et al. 1999) problem. In a force-free magnetic field, any electric currents are purely field aligned, and hence the Lorentz force $\boldsymbol{J} \times \boldsymbol{B}$, where $\boldsymbol{J}$ and $\boldsymbol{B}$ are the electric current density and the magnetic field vector, respectively, is zero by definition.

A force-free extrapolation presupposes that the boundary is force-free as well; otherwise, any quantitative correspondence between the actual and the calculated magnetic fields is compromised. Complex photospheric shearing motions, the large plasma density accompanied by a low ionization degree, the emergence of filamentary magnetic fields on a field-free photosphere, and the rapid rearrangements of the magnetic field topology in emerging flux regions make a force-free equilibrium unlikely. This was realized quite early (e.g., Krall et al. 1982). The magnetic "transition region" from forced to forcefree magnetic fields is thought to be located a few hundred kilometers above the photosphere (Metcalf et al. 1995), so the chromospheric magnetic field should be nearly force-free.

Since the study of Metcalf et al. (1995), the question of whether photospheric magnetic fields are force-free or not has become a focus of study. Berton (2000) applied a dimensional analysis to show that the magnetic forces may indeed be significant on the photosphere and proposed corrections to the conventional force-free extrapolations. Other works, however, suggest that the photospheric magnetic fields are not as far from a force-free equilibrium as previously thought. Moon et al. (2002) used a rigorous method to determine the magnetic forces (Aly 1984). They calculated the volume-integrated Lorentz force components, $F_{i}(i \equiv\{x, y, z\})$, and the integrated pressure force, $F_{0}$. In force-free fields, the ratios $\left(\left|F_{i}\right| / F_{0}\right)$ should all be much smaller than unity. Obviously, this method does not provide spatial information; the results are three spatially integrated, dimensionless quantities. Zhang (2001) attempted to decompose the electric current density into a "helical" and a "shear" component. Comparing the vertical shear and helical components (the only ones readily calculated by observables), he found a weaker shear component and concluded that most of the electric currents are due to helicity, so the magnetic configuration should be close to a force-free equilibrium. This approach has several problems. With only the $z$-components of the current density available, the decomposition is uncertain. Moreover, the helical current density cannot be field aligned in a purely force-free field unless the shear component is exactly zero; if the shear component is smaller but nonzero, the basic assumption of the method is inaccurate.

We demonstrate in this study a direct test of the assumption that the photospheric magnetic fields are force-free. In particular, we show that the vertical component $F_{z}$ of the Lorentz force can be calculated from single-height (i.e., photospheric) vector magnetic field measurements, provided that the vertical gradient, $\partial B / \partial z$, of the magnetic field strength $B$ is known. In our previous work (Georgoulis et al. 2004, hereafter Paper I), we have derived $\partial B / \partial z$ from observed vector magnetic fields, without resorting to an assumption of force-free fields. The test of the force-free condition and the implications for the flow of currents in the solar atmosphere are the subjects of this work. The paper is structured as follows: In $\S 2$ we derive $F_{z}$ analytically and we calculate the corresponding cross-field 
currents. In $\S 3$ we test the numerical accuracy of our method on analytical and extrapolated force-free magnetic fields. In $\S 4$ we apply the method to observed AR magnetic fields. In $\S 5$ we discuss the implications of our findings.

\section{LORENTZ FORCE, CROSS-FIELD CURRENTS, AND THE VERTICAL GRADIENT OF THE MAGNETIC FIELD}

\subsection{Vertical Component of the Lorentz Force}

The mean Lorentz force $\boldsymbol{F}$ exerted on a plasma occupying a given volume element is given by $\boldsymbol{F}=(1 / c) \boldsymbol{J} \times \boldsymbol{B}$, where $\boldsymbol{J}$ is the mean electric current density and $\boldsymbol{B}$ is the mean magnetic field vector at the volume element. In the limit of large conductivity, vector analysis can be used to decompose $\boldsymbol{F}$ into a magnetic tension term and a magnetic pressure term (e.g., Jackson 1962), namely,

$$
\boldsymbol{F}=\frac{1}{4 \pi}\left[(\boldsymbol{B} \cdot \nabla) \boldsymbol{B}-\frac{1}{2} \nabla B^{2}\right] .
$$

The three components $F_{m}$ ( $\left.m \equiv\{x, y, z\}\right)$, of the Lorentz force $\boldsymbol{F}$ can be then provided by equation (1) as follows:

$$
F_{m}=\frac{1}{4 \pi} \boldsymbol{B} \cdot \nabla B_{m}-B \frac{\partial B}{\partial m} .
$$

Alternatively, one can write

$F_{m}=\frac{B}{4 \pi}\left[\left(\frac{\partial B}{\partial m}\right)_{\mathrm{ff}}-\frac{\partial B}{\partial m}\right]$, where $\left(\frac{\partial B}{\partial m}\right)_{\mathrm{ff}}=\frac{1}{B} \boldsymbol{B} \cdot \nabla B_{m}$.

In the case of force-free magnetic fields, each of the above components of the Lorentz force is exactly zero, so $\partial B / \partial z=$ $(\partial B / \partial z)_{\mathrm{ff}}$. Unless the force-free assumption is explicitly adopted, however, none of the Lorentz force components can be assessed using solar magnetic field observations at a single altitude. The reason is that single-height observations preclude knowledge of the vertical derivative, $\partial / \partial z$, of any measured quantity. Nevertheless, let us consider the vertical component $F_{z}$ of the Lorentz force, given by

$$
F_{z}=\frac{B}{4 \pi} \frac{1}{B} \boldsymbol{B} \cdot \nabla B_{z}-\frac{\partial B}{\partial z}
$$

Provided that the $180^{\circ}$ ambiguity in the orientation of the transverse magnetic field vector has been resolved in a vector magnetogram, use of the divergence-free condition $\nabla \cdot \boldsymbol{B}=$ 0 for the magnetic field $\boldsymbol{B}$ provides the vertical derivative, $\partial B_{z} / \partial z$, of the vertical magnetic field $B_{z}$. Then, the vertical Lorentz force can be calculated from equation (4) if a reliable estimate of the vertical magnetic field gradient, $\partial B / \partial z$, is available. This expression cannot be a priori assumed to be equal or similar to $(\partial B / \partial z)_{\mathrm{ff}}$ (eq. [3]), since one then enforces the force-free hypothesis (as in the analysis of Cuperman et al. 1993). Our $\partial B / \partial z$ is derived in $\S 2.3$. Strong vertical Lorentz forces would invalidate the force-free hypothesis for the AR solar photosphere, while weak vertical Lorentz forces might be consistent with the force-free hypothesis. An additional concern, however, is how one discriminates between "strong" and "weak" Lorentz forces. We examine this issue in $\S 3$.

\subsection{Cross-Field Electric Current Density}

The field-aligned electric currents in a force-free field system are thought to represent significant free energy for powering solar flares and eruptive activity in general. If the field system is not force-free, then there are also cross-field electric currents. We need to know whether the cross-field currents are a significant addition to the free energy or are merely a curiosity. The knowledge of the vertical component $F_{z}$ of $\boldsymbol{F}$ leads to a partial knowledge of the cross-field electric current density in the magnetic configuration. This may be seen using two different geometrical approaches.

First, decompose the electric current density to two components, namely, $\boldsymbol{J}=\boldsymbol{J}_{\|}+\boldsymbol{J}_{\perp}$, where $\boldsymbol{J}_{\|}$and $\boldsymbol{J}_{\perp}$ are the fieldaligned and the cross-field component, respectively. Then, $\boldsymbol{F}=$ $(1 / c) \boldsymbol{J}_{\perp} \times \boldsymbol{B}$ and hence $\left|F_{z}\right|=(1 / c) J_{\perp_{h}} B_{h}|\sin \Delta \phi|$, where $J_{\perp_{h}}$ is the magnitude of the horizontal component $\boldsymbol{J}_{\perp_{h}}$ of $\boldsymbol{J}_{\perp}, B_{h}$ is the magnitude of the horizontal magnetic field $\boldsymbol{B}_{h}$, and $\Delta \phi$ is the angle formed between $\boldsymbol{J}_{\perp_{h}}$ and $\boldsymbol{B}_{h}$. This angle is obviously unknown, so $J_{\perp_{h}}$ cannot be calculated even if $F_{z}$ is known. Nevertheless, a lower limit $J_{\perp_{h(\min )}}$ of $J_{\perp_{h}}$ can be calculated for $|\sin \Delta \phi|=1$, namely,

$$
J_{\perp_{h(\min )}}=\frac{c\left|F_{z}\right|}{B_{h}} .
$$

The lower limit $J_{\perp_{h(\min )}}$ of $J_{\perp_{h}}$ also serves as a lower limit of the total cross-field current density, $J_{\perp}=\left(J_{\perp_{h}}^{2}+J_{\perp_{z}}^{2}\right)^{1 / 2}$, where $J_{\perp_{z}}$ is the vertical component of $\boldsymbol{J}_{\perp}: J_{\perp} \gtrsim J_{\perp_{h(\min )}}$.

Second, express the vertical Lorentz force as $\boldsymbol{F}_{z}=$ $(1 / c) \boldsymbol{J}_{h} \times \boldsymbol{B}_{h}$, where $\boldsymbol{J}_{h}$ is the horizontal component of the current density. Then, $\left|F_{z}\right|=(1 / c) J_{h_{p}} B_{h}$, where $J_{h_{p}}$ is the magnitude of the component $\boldsymbol{J}_{h_{p}}$ of $\boldsymbol{J}_{h}$, which is perpendicular to $\boldsymbol{B}_{h}$. It can be shown that $\boldsymbol{J}_{h_{p}}$ is also a component of the cross-field current density $\boldsymbol{J}_{\perp}$, since it lies on the cross section between the horizontal plane and the plane perpendicular to $\boldsymbol{B}$. Moreover, from equation (5) we note that $J_{h_{p}}=J_{\perp_{h(\min )}}$. Therefore, $J_{h_{p}}$ may also be viewed as a lower limit of the cross-field current density, $J_{\perp}$, although an analytical relation between $J_{\perp}$ and $J_{h_{p}}$ is not as straightforward as in case of $J_{\perp_{h}}$.

In the following we use equation (5) to calculate a minimum horizontal cross-field current density, $J_{\perp_{h(\min )}}$. The lower limit, $J_{\perp_{h(\min )}}$, of $J_{\perp_{h}}$ is hereafter denoted as $J_{\perp_{h}}$ for brevity. The only case for which no information on $F_{z}$ or $J_{\perp}$ is available occurs when $\boldsymbol{J}$ or $\boldsymbol{B}$ is purely vertical. In this case, $F_{z}=0$ by definition, so $J_{\perp_{h}}=0$ follows from equation (5).

If $F_{z} \neq 0$, on the other hand, one may translate a given vertical Lorentz force into a minimum requirement for the cross-field electric current density. Even a lower limit of the cross-field currents will be very helpful in testing the force-free hypothesis. A major issue, however, lies in the uncertainties associated with both the vertical Lorentz force and the minimum cross-field current density in the presence of observational noise in the measurements of the magnetic field components. This problem is dealt with by a complete error propagation analysis in $\S 3$ and in the Appendix.

\subsection{Vertical Gradient of the Magnetic Field Strength}

In Paper I we developed a structure minimization technique to resolve the $180^{\circ}$ ambiguity in solar vector magnetograms. The structure minimization approach calls for minimum crossfield (inhomogeneity) electric currents in Ampère's law. These currents occur because of cross-field inhomogeneities of the magnetic field strength. The inhomogeneity currents are reminiscent of the "sheath" currents thought to flow peripherally on the surface of magnetic flux tubes in the low solar atmosphere (e.g., Pizzo 1986; Ding et al. 1987; McClymont et al. 

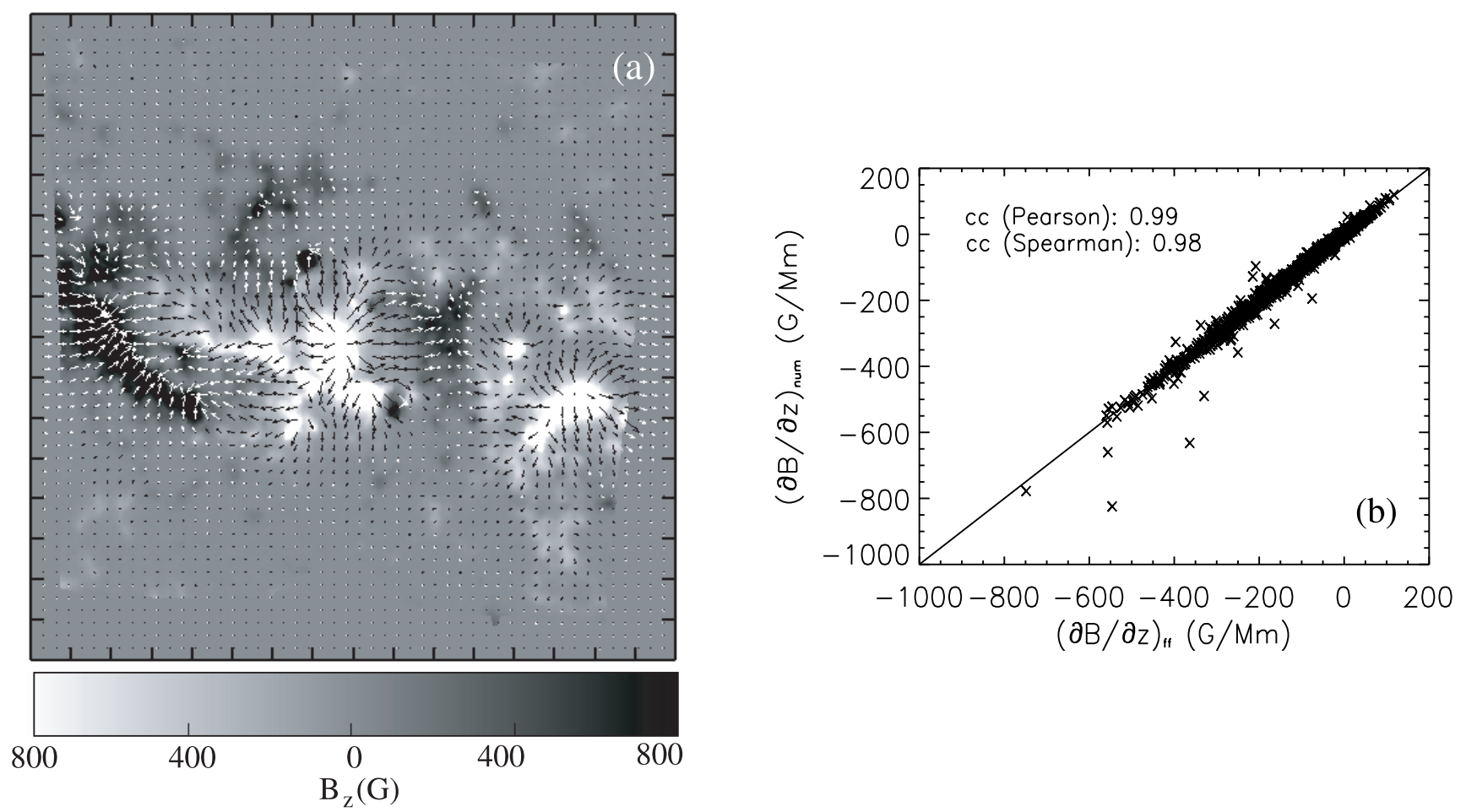

FIG. 1.-Testing our algorithm by means of a linear force-free approximation of observed AR magnetic fields. (a) The linear force-free magnetic field vector for NOAA AR 10030, calculated using a best-fit force-free parameter $\alpha_{\mathrm{AR}} \simeq 0.018 \mathrm{Mm}^{-1}$. A vector length equal to the tick mark separation corresponds to a horizontal field strength $\sim 1000 \mathrm{G}$. North is up; west is to the right. Tick mark separation is $20^{\prime \prime}$. (b) Comparison between the numerical and the expected vertical field gradients, namely, $(\partial B / \partial z)_{\text {num }}$ (eq. [7]) and $(\partial B / \partial z)_{\text {ff }}$ (eq. [3]), respectively. A random sample of 3000 pairs has been used. The straight line corresponds to $(\partial B / \partial z)_{\text {num }}=(\partial B / \partial z)_{\mathrm{ff}}$. The values of the linear (Pearson) and the nonparametric rank (Spearman) correlation coefficient (cc) are also provided. Comparison has been restricted to locations with $\left|B_{l}\right|>\delta B_{l}$ and $B_{\mathrm{tr}}>\delta B_{\mathrm{tr}}$ (for the IVM data, $\delta B_{l} \simeq 30 \mathrm{G}$ and $\delta B_{\mathrm{tr}} \simeq 60 \mathrm{G}$ ).

1997). Minimum inhomogeneity/sheath currents allow magnetic fields to be as space filling as possible. It is important to emphasize that the minimum structure approximation does not require force-free magnetic fields. The minimum structure vertical field gradient, $(\partial B / \partial z)_{p}$, is given by

$$
\left(\frac{\partial B}{\partial z}\right)_{p}=\frac{b_{z}}{b_{x}^{2}+b_{y}^{2}}\left(b_{x} \frac{\partial B}{\partial x}+b_{y} \frac{\partial B}{\partial y}\right),
$$

where $b_{i}=B_{i} / B(i \equiv\{x, y, z\})$ are the relative magnetic field components and the components of the unit vector $\hat{\boldsymbol{b}}$ along the magnetic field lines. The derived $(\partial B / \partial z)_{p}$ is a reliable estimate of the actual $\partial B / \partial z$, since it is found in Paper I to reproduce qualitatively the height variation of the photospheric magnetic fields, as described in rare cases where the total vertical field variation $d B / d z$ has been measured using nearly simultaneous multiheight (i.e., photospheric and chromospheric) magnetic field measurements (Leka \& Metcalf 2003).

\section{ANALYTICAL AND NUMERICAL TESTS}

Before applying the derivations of $\S 2$ to observed solar magnetic fields, it is important to verify their numerical accuracy and precision. This is done through the use of test cases, where the employed magnetic fields are a priori known to be force-free. These tests ensure that our results on observed magnetic fields are limited only by the quality of the observations.

We first consider an analytical force-free solution. Various linear and nonlinear force-free models have been generated by Low (1982) and have been further implemented by Low \& Lou (1990) and Cuperman et al. (1992, 1997). Applying our solution to some of these models, we verified that the analytical vertical gradient, $\partial B / \partial z$, calculated from the particular force-free solution and the expected vertical gradient, $(\partial B / \partial z)_{\text {fff }}$ (eq. [3]), coincide within numerical accuracy. Therefore, $F_{z}=0$ within numerical accuracy. Moreover, we calculated the ratio, $J_{\perp_{h}} /\left|J_{z}\right|$, of the minimum horizontal cross-field current density to the vertical current density, $J_{z}$. The values of the ratio were very small (median $\sim 1.2 \times 10^{-5}$; maximum $\sim 1 \times 10^{-2}$ ), differing from zero only because of roundoff errors. We conclude that the numerical accuracy of our method is satisfactory.

An analytical force-free solution is relatively simple and smooth. It is also important to test our algorithm for numerical force-free approximations of observed AR magnetic fields. The extrapolated fields are more complex and highly structured compared to analytical force-free fields, and the applied photospheric boundary condition is affected by observational noise. We utilize observations from the dynamic, complex NOAA AR 10030, observed by the Mees Solar Observatory's Imaging Vector Magnetograph (IVM; Mickey et al. 1996) on 2002 July 15 at 19:50 UT. We compute a linear force-free magnetic field using the photospheric (heliographic) vertical field as the boundary condition. The employed force-free parameter is $\alpha_{\mathrm{AR}} \simeq 0.018 \mathrm{Mm}^{-1}$ and was calculated by means of the "minimum residual" method of Leka \& Skumanich (1999); i.e., by minimizing the difference between the observed and the calculated horizontal magnetic fields. To perform this calculation, one needs to resolve the $180^{\circ}$ ambiguity in the AR. The ambiguity for this magnetogram has been resolved in Paper I.

The resulting force-free magnetic field, $\boldsymbol{B}_{\mathrm{ff}}$, is given in Figure $1 a$. Since $\boldsymbol{B}_{\mathrm{ff}}$ is fully known everywhere on the 

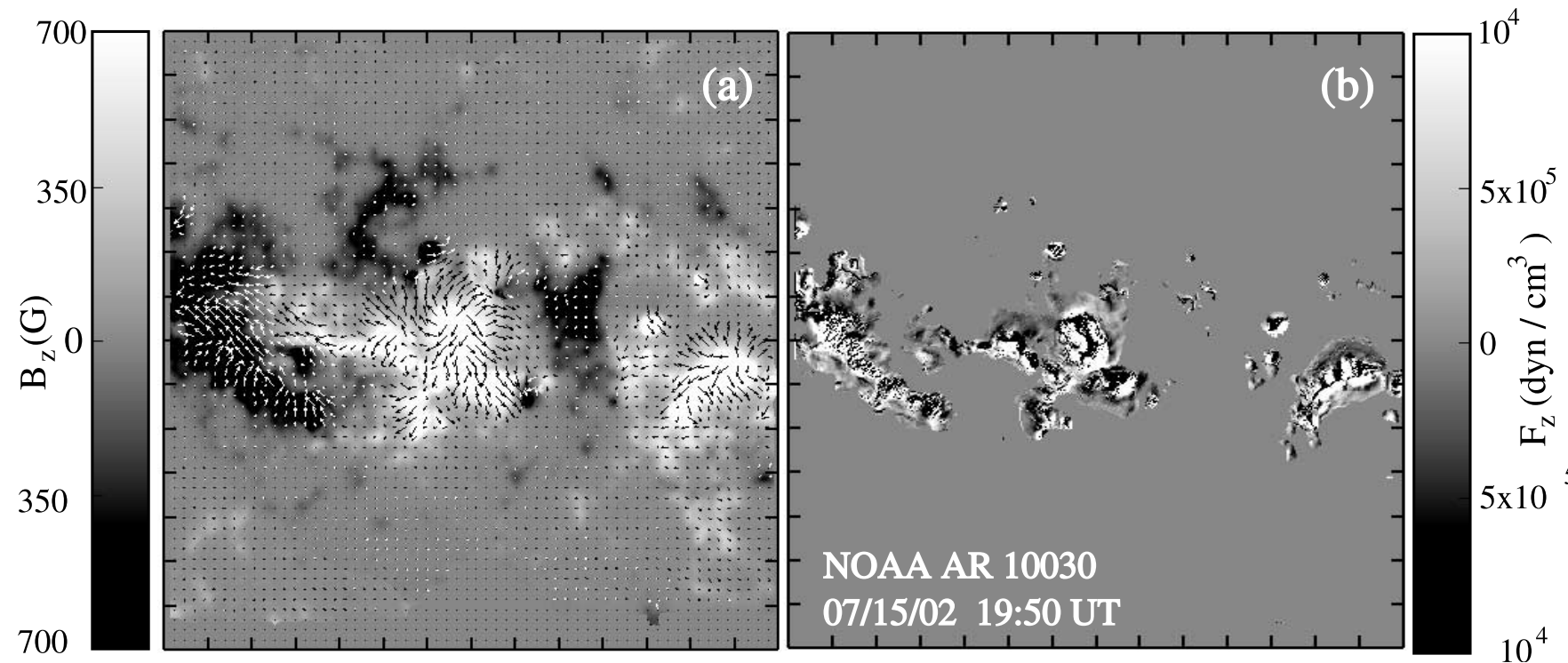

Fig. 2.-Calculation of the vertical Lorentz force in NOAA AR 10030 observed by IVM on 2002 July 15 at 19:50 UT. (a) Ambiguity-resolved horizontal magnetic field vector plotted on top of the vertical magnetic field in the AR. We show the heliographic magnetic field components on the image plane. A vector length equal to the tick mark separation corresponds to a horizontal field strength $\sim 1200 \mathrm{G}$. $(b)$ Vertical Lorentz force in the AR. Only areas where $\epsilon\left(F_{z}\right)<0.5$ are shown. Tick mark separation in both images is $20^{\prime \prime}$. North is up; west is to the right.

photosphere and above, one may either calculate analytically or evaluate numerically the vertical field gradient at any point on or above the photosphere. For simplicity, but also in order to test the numerical accuracy of our algorithm, we calculate numerically the vertical field gradient,

$$
\left(\frac{\partial B}{\partial z}\right)_{\operatorname{num}(z=d)} \simeq \frac{1}{2 d}\left[B_{\mathrm{ffr}(z=2 d)}-B_{\mathrm{ff}(z=0)}\right]
$$

where $d$ is an arbitrary height difference, and we compare it directly with the expected value, $\partial B / \partial z_{\mathrm{ff}(z=d)}$. Note that the comparison between the two vertical gradients takes place slightly above the photosphere $(z=d)$, rather than on $z=0$, because the force-free extrapolation is only applicable for $z \geq 0$. However, one may use arbitrarily small vertical steps $d$ in the force-free extrapolation, so the comparison between $(\partial B / \partial z)_{\mathrm{ff}}$ and $(\partial B / \partial z)_{\text {num }}$ occurs at heights $z$ asymptotically approaching zero.

The comparison between $(\partial B / \partial z)_{\text {num }}$ and the analytically expected $(\partial B / \partial z)_{\mathrm{ff}}$ is shown in Figure $1 b$. Evidently, $(\partial B / \partial z)_{\text {num }} \simeq$ $(\partial B / \partial z)_{\mathrm{ff}}$. The slope of the linear fit between the two gradients is indistinguishable from unity [the analytical relation $(\partial B / \partial z)_{\text {num }}=$ $(\partial B / \partial z)_{\text {ff }}$ is shown with the solid line], while the excellent quality of the fit is reflected on correlation coefficients $0.98-0.99$. We conclude that our method works well for observed AR magnetic fields if they are truly force-free.

\section{APPLICATION TO OBSERVED ACTIVE REGION MAGNETIC FIELDS}

We need to know whether the minimum-structure vertical field gradient ( $\S 2.3$ ), and subsequently the vertical Lorentz force and the minimum cross-field current density $(\S \S 2.1$ and 2.2 , respectively), can be calculated in the presence of observational noise. The calculations are performed on the observer's (image) plane using the heliographic magnetic field components and are accompanied by an error propagation analysis. The details of this analysis are discussed in the Appendix, but the idea is to introduce typical uncertainties $\delta B_{l}$, $\delta B_{\mathrm{tr}}$, and $\delta \phi_{l}$, for the (line of sight) longitudinal field, $B_{l}$, the transverse field, $B_{\mathrm{tr}}$, and the azimuth, $\phi_{l}$, of $\boldsymbol{B}_{\mathrm{tr}}$, respectively, into the geometrical transformation to the heliographic reference system (Gary \& Hagyard 1990). The outcome is the calculation of the uncertainties $\delta B_{z}$ and $\delta B_{h}$ of the (heliographic) vertical field, $B_{z}$, and horizontal field, $B_{h}$, respectively, which are then used to provide the uncertainties in the estimation of the minimum structure vertical field gradient, $(\partial B / \partial z)_{p}$ (eq. [6]), the vertical Lorentz force, $F_{z}$ (eq. [4]), and the minimum cross-field current density, $J_{\perp_{h}}$ (eq. [5]). In the following, we use IVM vector magnetograms of the AR photosphere. Typical IVM line-of-sight uncertainties, as they occur from the fitting uncertainties of the observed Stokes profiles, are $\delta B_{l} \simeq 30 \mathrm{G}, \delta B_{\text {tr }} \simeq 60 \mathrm{G}$, and $\delta \phi_{l} \simeq 2^{\circ}$.

\subsection{NOAA AR 10030}

In Figure $2 a$ we show the minimum structure ambiguity-free magnetic field vector for NOAA AR 10030, as reconstructed in Paper I. The magnetic field vector consists of the heliographic magnetic field components on the image plane. The respective vertical Lorentz force is shown in Figure $2 b$, in which we include only areas where the relative error $\epsilon\left(F_{z}\right)=\left(\delta F_{z} /\left|F_{z}\right|\right)$ (with $\delta F_{z}$ being the uncertainties in $F_{z}$; see the Appendix) is less than $0.5(50 \%)$. In the following, we accept this maximum value of the relative error as our tolerance limit in the calculation of averages and means. This selection of the relative error $\epsilon\left(F_{z}\right)$ represents $63 \%$ of the total well-measured magnetic flux in the AR. By "well-measured" magnetic flux, we mean the magnetic flux calculated in locations where $\left|B_{l}\right|>\delta B_{l}$ and $B_{\mathrm{tr}}>\delta B_{\mathrm{tr}}$. From Figure $2 b$, we note that $\epsilon\left(F_{z}\right)<0.5$ for the most interesting strong-field areas of the AR. We obtain strong Lorentz forces and, as seen below, powerful cross-field currents in these areas. Figure $2 b$ also shows that it is difficult to assess a reliable estimate of $F_{z}$ in strong-field plages, so our conclusions will be limited to sunspots, pores, and strong-field regions surrounding these areas. Apparently, the remaining $37 \%$ of the total flux for which $\epsilon\left(F_{z}\right)>0.5$ consists primarily of plage magnetic fields. The lack of information in plages is due to their distinctive magnetic field geometry, as we discuss 

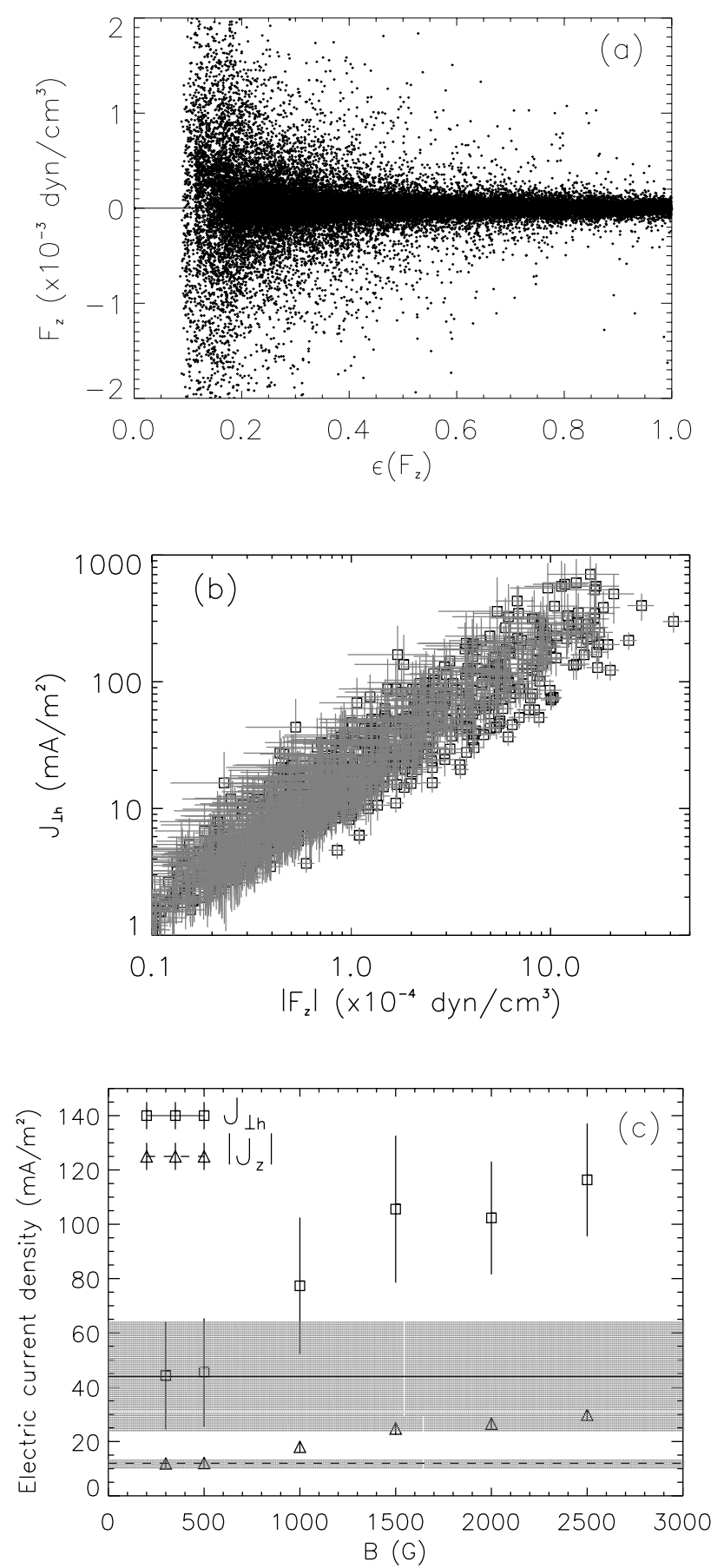

FIG. 3.-Vertical Lorentz force and the minimum cross-field electric current density in NOAA AR 10030. (a) Vertical Lorentz force $F_{z}$ vs. the respective relative error $\epsilon\left(F_{z}\right)$. (b) Correlation between the vertical Lorentz force and the respective minimum cross-field current density. Both the error bars of $\left|F_{z}\right|$ (horizontal lines) and of $J_{\perp_{h}}$ (vertical lines) are shown. (c) Correlation between the magnetic field strength, the mean values of the minimum cross-field current density, and the mean vertical current density. The magnetic field values correspond to the minimum values of each sample, while the points and the error bars correspond to the mean $J_{\perp_{h}}$ (rectangles) and $\left|J_{z}\right|$ (triangles) of each sample. The overall mean $\bar{J}_{\perp_{h}}\left(\left|\bar{J}_{z}\right|\right)$ is indicated by a solid (dashed) line, while the shaded area surrounding the line indicates the uncertainty associated with the overall mean. The various samples in $(b)$ and $(c)$ have been selected for $\epsilon\left(F_{z}\right)<0.5$. in $\S 5$. For $\epsilon\left(F_{z}\right)<0.5$, the mean absolute vertical Lorentz force in the AR is $\left|\bar{F}_{z}\right|=(2.2 \pm 0.7) \times 10^{-4} \mathrm{dyn} \mathrm{cm}^{-3}$.

From Figure $3 a$ we note that the solution for $\left|F_{z}\right|$ is well behaved: the inferred values are small in locations where the relative error is large. Strong $\left|F_{z}\right|$ are obtained for $\epsilon\left(F_{z}\right) \lesssim 0.4$. This implies that the large Lorentz forces are not an artifact attributable to observational noise but should be considered real. It also implies that if we imposed a more stringent upper limit for $\epsilon\left(F_{z}\right)$, we would obtain larger $\left|\bar{F}_{z}\right|$ associated with smaller uncertainties.

In Figure $3 b$ we relate the vertical Lorentz force with the respective minimum cross-field current density $J_{\perp_{h}}$. The data points correspond to a random sample of $1000\left(\left|F_{z}\right|, J_{\perp_{h}}\right)$ pairs for which $\epsilon\left(F_{z}\right)<0.5$. The calculated uncertainties of both $F_{z}$ and $J_{\perp_{h}}$ are also shown in the plot. We note that strong Lorentz forces give rise to similarly strong cross-field currents. This relation is well described by a power law, and an empirical logarithmic scaling between $J_{\perp_{h}}$ and $F_{z}$ reads

$$
\begin{aligned}
\log \left[J_{\perp_{h}}\left(\mathrm{~mA} \mathrm{~m}^{-2}\right)\right] \simeq & (1.01 \pm 0.002) \\
& \times \log \left[\left|F_{z}\right|\left(\mathrm{dyn} \mathrm{cm}^{-3}\right)\right]+5.31
\end{aligned}
$$

Figure $3 b$ shows significant cross-field electric currents densities, typically several tens to $100 \mathrm{~mA} \mathrm{~m}^{-2}$ and, rarely, several times $100 \mathrm{~mA} \mathrm{~m}^{-2}$. The mean value of the minimum possible cross-field current density is $\bar{J}_{\perp_{h}}=44 \pm 20 \mathrm{~mA} \mathrm{~m}{ }^{-2}$. Had we used an upper limit of, say, 0.2 for $\epsilon\left(F_{z}\right)$, we would have obtained a mean cross-field current density equal to $75 \pm$ $18 \mathrm{~mA} \mathrm{~m}^{-2}$, but this would represent only $\sim 20 \%$ of the wellmeasured flux in the AR. These large strong-field currents should be considered evidence that the photospheric magnetic configuration in NOAA AR 10030 was not force-free at the time of our study. This conclusion is further established in Figure $3 c$, where we show the mean cross-field electric current density as a function of the magnetic field strength in the AR. The values of the magnetic field in the abscissa correspond to the minimum magnetic fields with which each sample was selected. To provide a context for the values of the cross-field electric current density, $J_{\perp_{h}}$, we also include in Figure $3 c$ the respective means of the absolute value of the vertical current density, $J_{z}=(\nabla \times \boldsymbol{B})_{z}$, and their uncertainties. The overall means $\bar{J}_{\perp_{h}}$ and $\left|\bar{J}_{z}\right|$ are given by the solid and the dashed straight lines, respectively, while the respective uncertainties are indicated by the shaded areas surrounding the straight lines. We obtain $\left|\bar{J}_{z}\right|=12 \pm 1.7 \mathrm{~mA} \mathrm{~m}^{-2}$. Figure $3 c$ shows two important results: First, both $J_{\perp_{h}}$ and $\left|J_{z}\right|$ become stronger as the magnetic fields in the AR become stronger. The overall mean values are typically found in magnetic fields $B \lesssim 500 \mathrm{G}$. Second, $\bar{J}_{\perp_{h}}$ is always $\sim 3-4$ times larger than $\left|\bar{J}_{z}\right|$. This is further evidence that the photospheric magnetic fields in NOAA AR 10030 are forced. The calculated values of $J_{z}$ are in line with several previous studies that have shown that $J_{z}$ is on the order of a few to a few times $10 \mathrm{~mA} \mathrm{~m}^{-2}$ on the AR photosphere (de La Beaujardière et al. 1993; Metcalf et al. 1994; Gary \& Démoulin 1995; Leka et al. 1996; Li et al. 1997; and others).

NOAA AR 10030 is admittedly a dynamic region, seat of a strong X3 flare approximately 15 minutes after the magnetogram of Figure $2 a$ was taken (Liu et al. 2003; Gary \& Moore 2004). This suggests that a complex, forced magnetic configuration in the AR photosphere should probably be expected. In the following, we study a magnetogram taken from a nonflaring AR. 


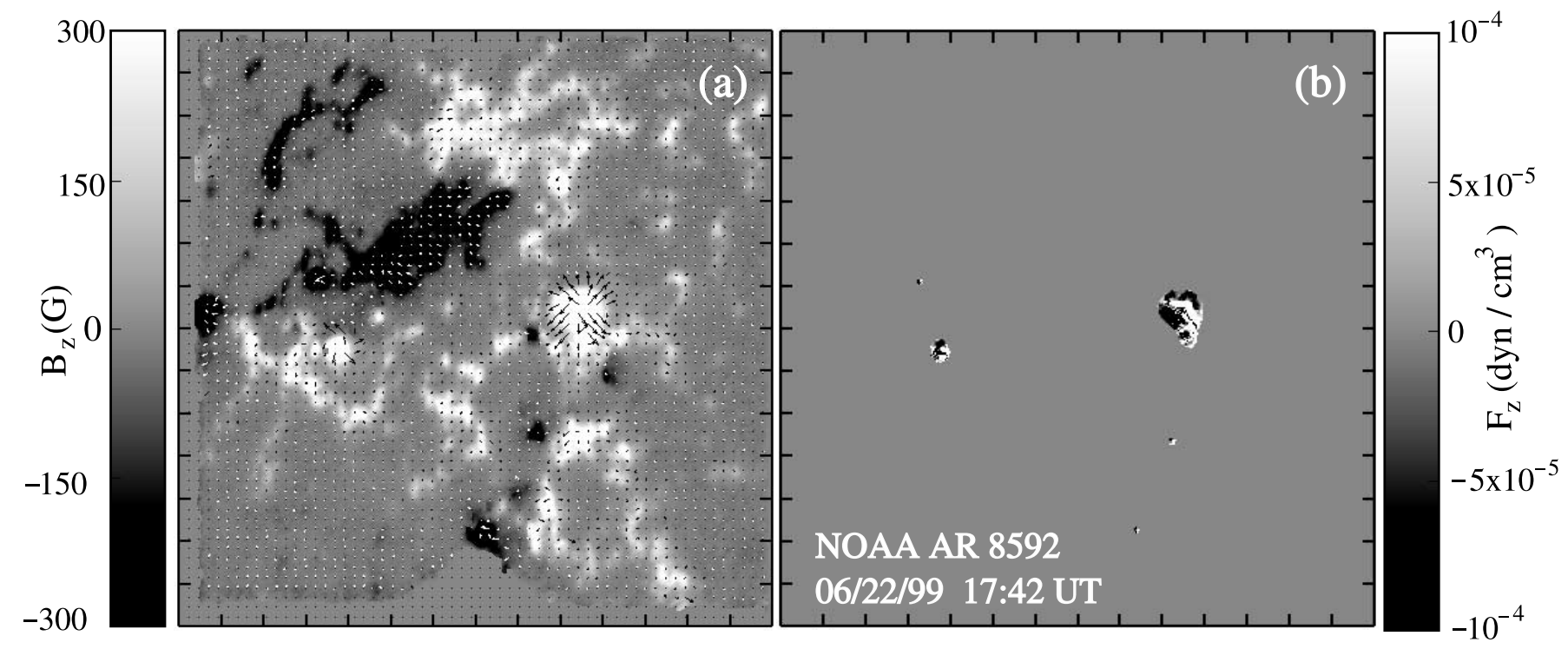

FIG. 4.-Calculation of the vertical Lorentz force in NOAA AR 8592 observed by IVM on 1999 June 22 at 17:42 UT (a) Ambiguity-resolved horizontal magnetic field vector plotted on top of the vertical magnetic field in the AR. We show the heliographic magnetic field components on the image plane. A vector length equal to the tick mark separation corresponds to a horizontal field strength $\sim 700 \mathrm{G}$. $(b)$ Vertical Lorentz force in the AR. Only areas where $\epsilon\left(F_{z}\right)<0.5$ are shown. Tick mark separation in both images is $20^{\prime \prime}$. North is up; west is to the right.

\subsection{NOAA AR 8592}

NOAA AR 8592 was a quiescent, extended region, and was observed by IVM on 1999 June 22. Using linear force-free extrapolations, LaBonte (2002) concluded qualitatively that the photospheric magnetic configuration in this AR is forced. In Figure $4 a$ we show the minimum structure, ambiguity-free, heliographic magnetic field vector of the AR on the image plane. This vector was reconstructed in Paper I. We noticed in $\S 4.1$ that it is difficult to perform a reliable calculation of $F_{z}$ and $J_{\perp_{h}}$ in plages. This is shown dramatically in the case of NOAA AR 8592 , which is a region predominantly consisting of several extended strong-field plages. The calculated vertical Lorentz force for $\epsilon\left(F_{z}\right)<0.5$ is shown in Figure $4 b$ and represents only $20 \%$ of the total well-measured flux in the AR. The only areas in which $F_{z}$ is reliably calculated are a small sunspot and a number of small pores. We will study these areas in an attempt to investigate whether isolated, quiescent sunspots and pores are force-free or not. The mean absolute vertical Lorentz force in the AR is $\left|\bar{F}_{z}\right|=(2.2 \pm 0.6) \times 10^{-4} \mathrm{dyn} \mathrm{cm}^{-3}$. Note the close correspondence in $\left|\bar{F}_{z}\right|$ for NOAA ARs 10030 and 8592 , despite the profound morphological differences between the two ARs.

The vertical Lorentz force and its relative error are given in Figure $5 a$. The revealed behavior is qualitatively the same as that of NOAA AR 10030 , namely, that $\epsilon\left(F_{z}\right)$ is typically below 0.4 for large values of $\left|F_{z}\right|$. Therefore, these values of the vertical Lorentz force in the AR are not an artifact of the observational noise. The minimum horizontal cross-field current density and the vertical Lorentz force are correlated in Figure $5 b$, in which we show 1000 randomly selected $\left(\left|F_{z}\right|, J_{\perp_{h}}\right)$ pairs for which $\epsilon\left(F_{z}\right)<0.5$. Again, we find that large vertical Lorentz forces typically correspond to strong cross-field currents and that the relation is well described by a power law. An empirical scaling between $\left|F_{z}\right|$ and $J_{\perp_{h}}$ reads

$$
\begin{aligned}
\log \left[J_{\perp_{h}}\left(\mathrm{~mA} \mathrm{~m}^{-2}\right)\right] \simeq & (0.96 \pm 0.006) \\
& \times \log \left[\left|F_{z}\right|\left(\mathrm{dyn} \mathrm{cm}^{-3}\right)\right]+5.02 .
\end{aligned}
$$

This scaling law is similar to that found for NOAA AR 10030 , with NOAA AR 8592 implying somewhat weaker currents. For example, for $\left|F_{z}\right|=10^{-4}$ dyn cm $\mathrm{cm}^{-3}$ we find $J_{\perp_{h}} \simeq 18.6 \pm$ 0.3 and $J_{\perp_{h}} \simeq 15.2 \pm 0.9 \mathrm{~mA} \mathrm{~m}^{-2}$ for NOAA ARs 10030 and 8592, respectively. For $\left|F_{z}\right|=5 \times 10^{-4}$ dyn cm ${ }^{-3}$, the respective values are $J_{\perp_{h}} \simeq 94.6 \pm 1.4$ and $J_{\perp_{h}} \simeq 71 \pm 3.2 \mathrm{~mA} \mathrm{~m}^{-2}$. The overall mean for the minimum cross-field current density in the AR is $\bar{J}_{\perp_{h}}=34 \pm 14 \mathrm{~mA} \mathrm{~m}^{-2}$. As a result, the photospheric magnetic fields in NOAA AR 8592 should also be considered forced. The non-force-free character of NOAA AR 8592 is exemplified in Figure $5 c$, where we show the mean cross-field current densities and the mean vertical current densities for various subsets of the magnetic field strength. Figure $5 c$ indicates that $\bar{J}_{\perp_{h}}$ and $\left|\bar{J}_{z}\right|$ become stronger as the magnetic field increases and that $\bar{J}_{\perp_{h}}$ is typically 2-3 times larger than $\left|\bar{J}_{z}\right|$. The mean values of $J_{\perp_{h}}$ and $\left|J_{z}\right|$ are found in magnetic fields $B \lesssim$ $500 \mathrm{G}$, as in NOAA AR 10030. The overall mean for the absolute vertical current density is $\left|\bar{J}_{z}\right|=13.8 \pm 1.6 \mathrm{~mA} \mathrm{~m}^{-2}$.

In summary, the behavior of the vertical Lorentz force and the cross-field current density in the quiescent NOAA AR 8592 are found to be very similar to the respective behavior in the dynamic NOAA AR 10030. Based on the mean quantities, the magnetized photosphere of NOAA AR 8592 is also non-forcefree, although less so compared to NOAA AR 10030. The "degree of non-force-freeness" that enables comparison between the two ARs is quantified by (1) the overall means of $\left|F_{z}\right|$ and $J_{\perp_{h}}$ for a given relative error and (2) the ratio $\left(\bar{J}_{\perp_{h}} /\left|\bar{J}_{z}\right|\right)$. We emphasize again that our results do not apply to plages, for which the relative errors are too large to permit a reliable calculation. Based on our analysis, the force-free hypothesis cannot be ruled out in plages, although it is not obvious why the conditions in these areas should be substantially different than elsewhere in the AR.

\subsection{Improving the Signal-to-Noise Ratio: NOAA AR 9114}

A way to investigate how the quality of the observations affects our calculations and conclusions is to improve the signal-to-noise ratio. This is done by averaging over several 

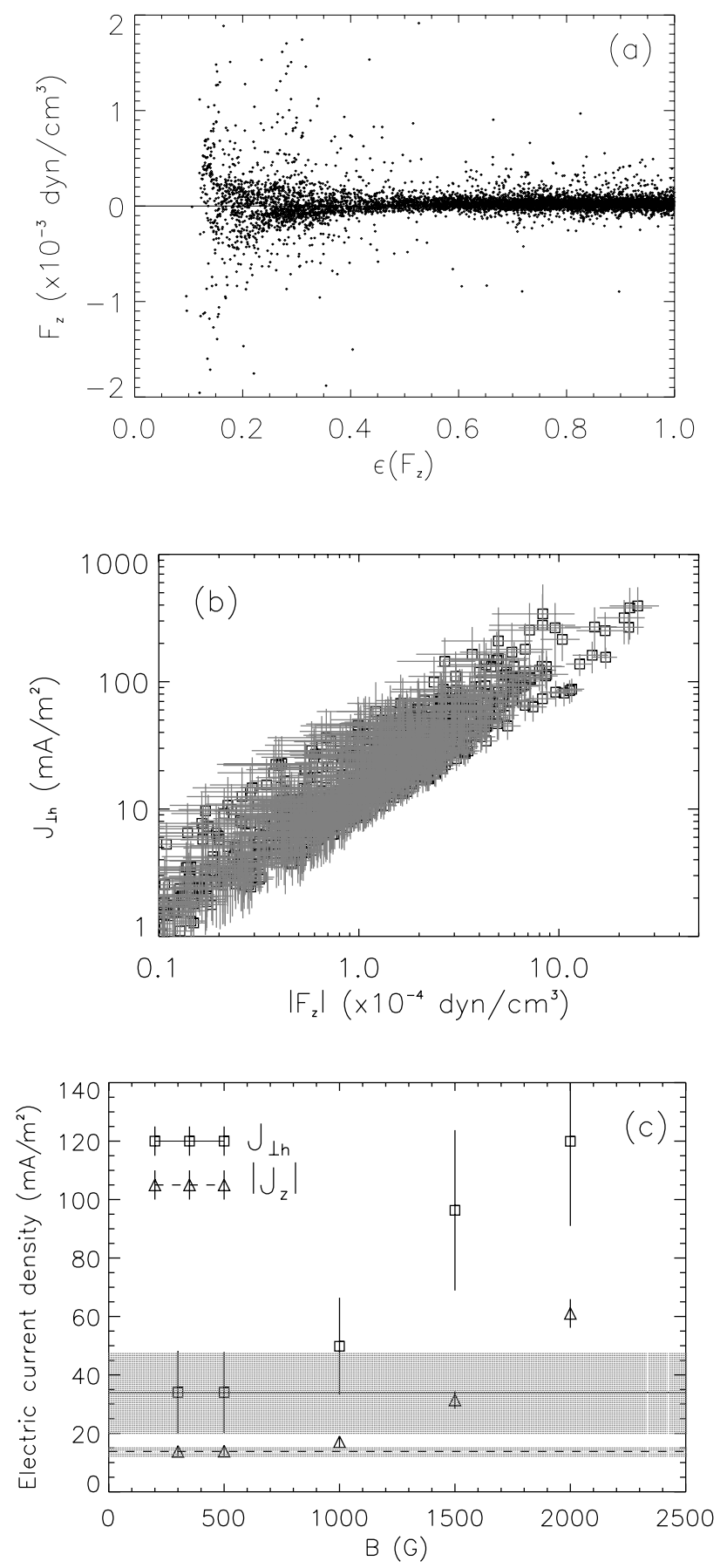

FIG. 5.-Vertical Lorentz force and the minimum cross-field electric current density in NOAA AR 8592. (a) Vertical Lorentz force $F_{z}$ vs. the respective relative error $\epsilon\left(F_{z}\right)$. (b) Correlation between the vertical Lorentz force and the respective minimum cross-field current density. Both the error bars of $\left|F_{z}\right|$ (horizontal lines) and of $J_{\perp_{h}}$ (vertical lines) are shown. (c) Correlation between the magnetic field strength, the mean values of the minimum cross-field current density, and the mean vertical current density. The magnetic field values correspond to the minimum values of each sample, while the points and the error bars correspond to the mean $J_{\perp_{h}}$ (rectangles) and $\left|J_{z}\right|$ (triangles) of each sample. The overall mean $\bar{J}_{\perp_{h}}\left(\left|\bar{J}_{z}\right|\right)$ is indicated by a solid (dashed) line, while the shaded area surrounding the line indicates the uncertainty associated with the overall mean. The various samples in $(b)$ and $(c)$ have been selected for $\epsilon\left(F_{z}\right)<0.5$.

magnetograms of a given AR. Averaging results in smaller uncertainties for the magnetic field components. The underlying assumption is that the magnetic topology in the AR does not change significantly in the magnetograms used for the averaging. Averaging is thus viewed as the equivalent of re-

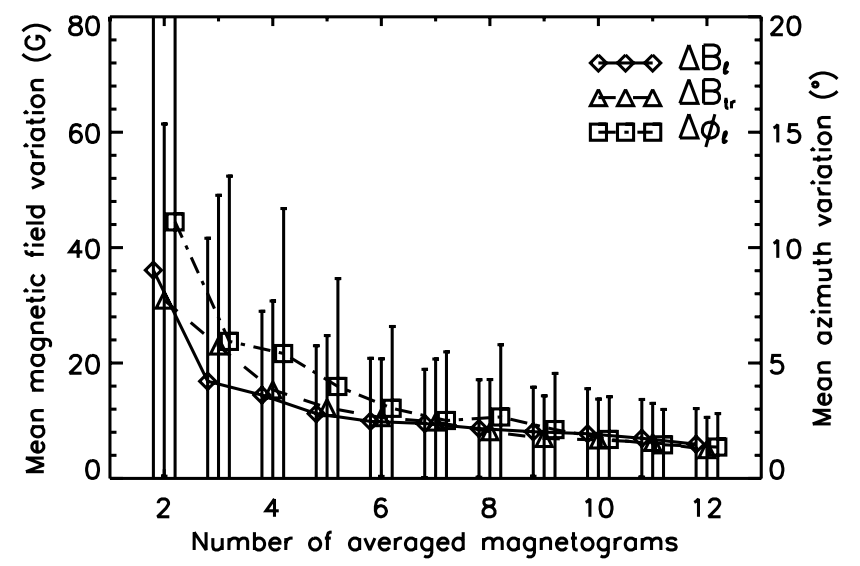

FIG. 6.- Improving the signal-to-noise ratio by averaging over several magnetograms of NOAA AR 9114. Shown are the mean differences, $\Delta B_{l}$, in the longitudinal magnetic field (diamonds; solid line), $\Delta B_{\mathrm{tr}}$ in the transverse magnetic fields (triangles; dashed line), and $\Delta \phi_{l}$ in the azimuth angle of $\boldsymbol{B}_{\mathbf{t r}}$ (rectangles; dash-dotted line) between two averaged magnetograms $\boldsymbol{B}_{1}$ and $\boldsymbol{B}_{2}$ comprising $m$ and $m+1$ individual magnetograms, respectively. The error bars represent the standard deviations for each array of differences.

peatedly measuring the same magnetic fields to decrease the level of uncertainties at every strong field location.

We have averaged several magnetograms of NOAA AR 9114, observed by IVM on 2000 August 8. Before averaging, (1) the $180^{\circ}$ ambiguity has been resolved for each of the magnetograms (as described in Paper I) and (2) the magnetograms have been coaligned. The improvement of the signal-to-noise ratio as a function of the number of magnetograms used in the averaging in given in Figure 6 . The data points refer to the mean differences $\Delta B_{l}, \Delta B_{\mathrm{tr}}$, and $\Delta \phi_{l}$ in the longitudinal field, $B_{l}$, the transverse field, $B_{\text {tr }}$, and the azimuth, $\phi_{l}$, of $\boldsymbol{B}_{\text {tr }}$, respectively, between two averaged magnetograms $\boldsymbol{B}_{1}$ and $\boldsymbol{B}_{2}$, where $\boldsymbol{B}_{1}$ is an average over $m$ individual magnetograms and $\boldsymbol{B}_{2}$ is an average over $m+1$ magnetograms ( $m$ being an arbitrary integer). The uncertainties are the standard deviation of each array of differences calculated as the square root of the variance in each array. These uncertainties may include actual signal changes owning to the evolution of the magnetic field vector in the AR. We assume that this signal is of small amplitude. From Figure 6 we note that both the mean changes and the associated uncertainties decrease sharply as the number of magnetograms involved in the averaging increases.

In Figure $7 a$ we show the average heliographic magnetic field vector in the AR, constructed from 12 magnetograms obtained between 17:39 UT and 21:47 UT on 2000 August 8. The difference between $\boldsymbol{B}_{m=12}$ and $\boldsymbol{B}_{m=11}$ has provided the uncertainties $\delta B_{l}, \delta B_{\text {tr }}$, and $\delta \phi_{l}$ independently at each location in the AR. In the previous cases ( $\delta \S 4.2$ and 4.3 ), the above uncertainties were unique numbers for the entire magnetogram and were obtained by the fitting of the observed Stokes profiles. An alternative in the case of an averaged magnetogram would be to average over several Stokes profiles and to fit the average profile, thus obtaining again unique estimates for $\delta B_{l}, \delta B_{\mathrm{tr}}$, and $\delta \phi_{l}$. These estimates will certainly be smaller than those obtained from a single magnetogram. In our case, the error analysis of the Appendix was performed using different $\delta B_{l}, \delta B_{\text {tr }}$, and $\delta \phi_{l}$ for each location.

The vertical Lorentz force associated with the average magnetogram of Figure $7 a$ is shown in Figure $7 b$ for $\epsilon\left(F_{z}\right)<0.5$. This choice now represents $99.9 \%$ of the well-measured magnetic flux in the AR, implying that averaging has decreased the 

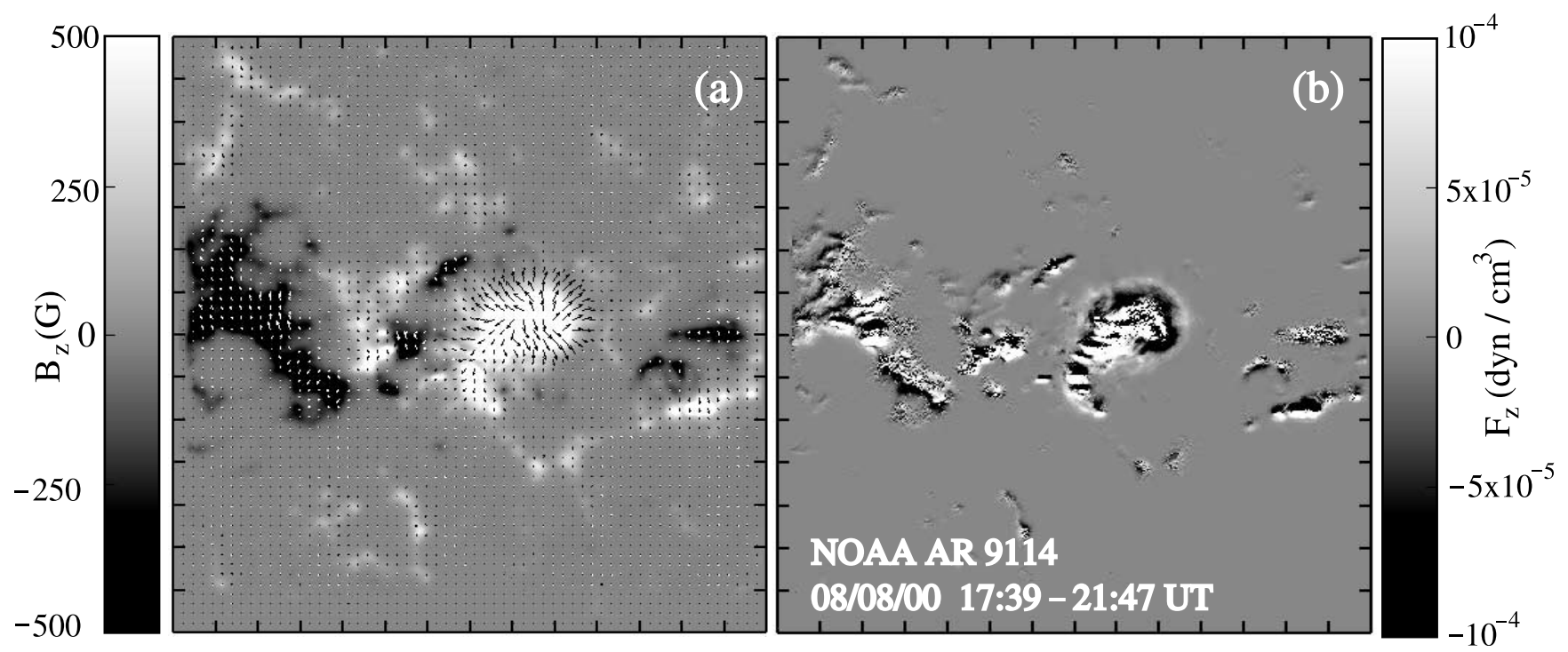

FIG. 7. - Calculation of the vertical Lorentz force for an average of 12 magnetograms of NOAA AR 9114 observed by IVM on 2000 August 8 between 17:39 UT and 21:47 UT. (a) Average of the ambiguity-resolved horizontal magnetic field vector plotted on top of the average vertical magnetic field. A vector length equal to the tick mark separation corresponds to a horizontal field strength $\sim 1200 \mathrm{G}$. (b) Corresponding vertical Lorentz force. Only areas where the relative error $\epsilon\left(F_{z}\right)<0.5$ are shown. Tick mark separation in both images is $20^{\prime \prime}$. North is up; west is to the right.

uncertainties dramatically. Some small plages appear in the image of Figure $7 b$, besides sunspots and surrounding areas. The vertical Lorentz force in plages is generally smaller than that of sunspots, on the order of a few times $10^{-5}$ dyn $\mathrm{cm}^{-3}$. However, as we explain in $\S 5$, the peculiar magnetic field geometry in plages allows strong cross-field horizontal currents even with moderate vertical Lorentz forces. As for the rest of the AR, averaging allows us to use weaker magnetic fields in the calculations, which leads to a decrease of the mean absolute vertical Lorentz force. This is now $\left|\bar{F}_{z}\right|=(0.24 \pm 0.02) \times$ $10^{-4}$ dyn $\mathrm{cm}^{-3}$.

Figure $8 a$ also shows that the statistical behavior of $\left|F_{z}\right|$ and its errors improves significantly when averaging over a number of magnetograms. The relative error $\epsilon\left(F_{z}\right)$ almost exclusively stays below 0.3. In Figure $8 b$ we have chosen a random sample of $1000\left(\left|F_{z}\right|, J_{\perp_{h}}\right)$ pairs for $\epsilon\left(F_{z}\right)<0.5$. The decreased Lorentz forces of the averaged magnetogram are also shown here, and they generally trigger smaller cross-field current densities. Again, a power-law fit describes the relation between $\left|F_{z}\right|$ and $J_{\perp_{h}}$ and yields

$$
\begin{aligned}
\log \left[J_{\perp_{h}}\left(\mathrm{~mA} \mathrm{~m}^{-2}\right)\right] \simeq & (0.8 \pm 0.0002) \\
& \times \log \left[\left|F_{z}\right|\left(\mathrm{dyn} \mathrm{cm}^{-3}\right)\right]+5.14 .
\end{aligned}
$$

This scaling law is much steeper compared to the scaling laws found in the single magnetograms of NOAA ARs 10030 and 8592. For example, for $\left|F_{z}\right|=10^{-4} \mathrm{dyn} \mathrm{cm}^{-2}$, equation (10) gives $J_{\perp_{h}} \simeq 87.1 \pm 0.2 \mathrm{~mA} \mathrm{~m}^{-2}$, as opposed to $18.6 \pm 0.3$ and $15.2 \pm 0.9 \mathrm{~mA} \mathrm{~m}^{-2}$ for NOAA ARs 10030 and 8592, respectively. For $\left|F_{z}\right|=5 \times 10^{-4}$ dyn cm $\mathrm{cm}^{-2}$, equation (10) gives $315 \pm$ $0.5 \mathrm{~mA} \mathrm{~m}{ }^{-2}$. Because the uncertainties are small in the averaged magnetogram, which enables us to involve weaker magnetic fields in the calculation, however, we find a much weaker mean Lorentz force, which corresponds to a smaller mean crossfield current density. We find $\bar{J}_{\perp_{h}}=12.1 \pm 1.65 \mathrm{~mA} \mathrm{~m}^{-2}$. This is smaller than the means in both NOAA AR 10030 and 8592, even under a steeper scaling law between $\left|F_{z}\right|$ and $J_{\perp_{h}}$.

In Figure $8 c$, we provide the averages of $\left|F_{z}\right|$ and $J_{\perp_{h}}$ for different magnetic field subsets. We note that the overall means are now found in $B \lesssim 100-200 \mathrm{G}$, as opposed to $B \lesssim 500 \mathrm{G}$ in NOAA ARs 10030 and 8592 . This is also a consequence of the smaller uncertainties and the weaker magnetic fields used in the calculations in the averaged magnetogram. However, the mean vertical current density is now 4-7 times smaller than the mean cross-field current density, showing an overall mean $\left|\bar{J}_{z}\right|=3.1 \pm 0.1 \mathrm{~mA} \mathrm{~m}^{-2}$. We conclude that, despite smaller values of the Lorentz forces and respective cross-field current densities, the non-force-free character of the photospheric magnetic fields as quantified by the ratio $\bar{J}_{\perp_{h}} /\left|\bar{J}_{z}\right|$ is actually enhanced, rather than suppressed, in the averaged magnetogram. In other words, one may decrease the mean values of the Lorentz forces and the cross-field currents when averaging over several magnetograms, but the essential properties of the non-force-free magnetic fields in the AR photosphere are still present and cannot be smoothed away by averaging.

Our results for the overall mean values $\left|\bar{F}_{z}\right|, \bar{J}_{\perp_{h}}$, and $\left|\bar{J}_{z}\right|$ in the three subject ARs are summarized for comparison in Table 1.

\section{DISCUSSION AND CONCLUSIONS}

Using photospheric vector magnetograms, we estimate the vertical Lorentz force and a corresponding lower limit of the cross-field electric current density in solar active regions. To perform this calculation, (1) the vertical gradient, $\partial B / \partial z$, of the magnetic field strength must be known and (2) the $180^{\circ}$ ambiguity in the magnetogram must be resolved. The numerical accuracy of our method is found to be satisfactory in both theoretical and approximated force-free solutions. Hence, our results are only limited by the quality of the observations and the reliability of the employed $\partial B / \partial z$.

Direct calculation of $\partial B / \partial z$ implies knowledge of both photospheric and chromospheric magnetic fields in an AR. Comparing photospheric and chromospheric magnetograms is not a routine process, however, mostly because of the very limited availability of the latter but also because of simultaneity and coalignment issues in case multiheight magnetograms are available. Therefore, a reliable, easily obtained estimate of $\partial B / \partial z$ can be very useful. The structure minimization method, introduced in Paper I, provides an approximation of the 

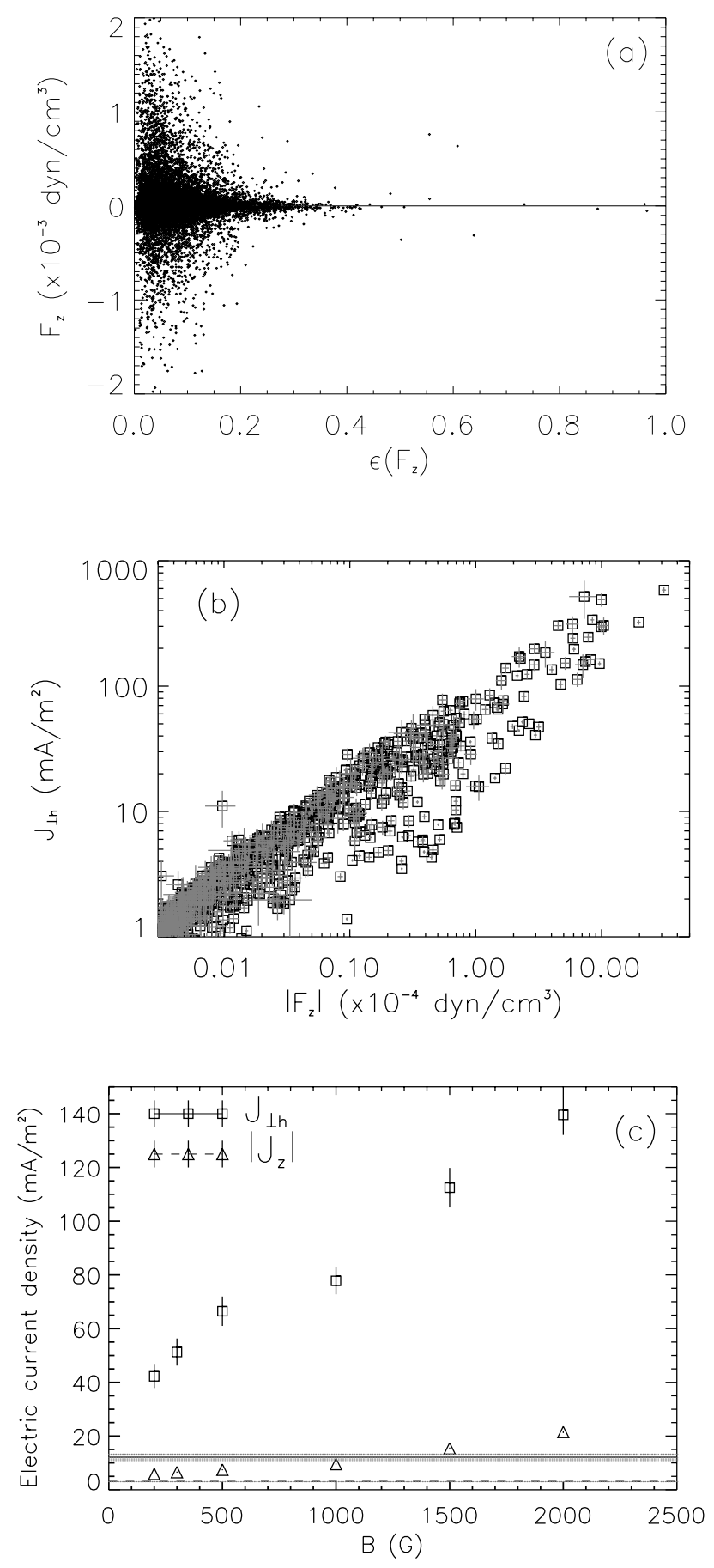

FIG. 8.-Vertical Lorentz force and the minimum cross-field electric current density in the averaged magnetogram of NOAA AR 9114. (a) Vertical Lorentz force $F_{z}$ vs. the respective relative error $\epsilon\left(F_{z}\right)$. (b) Correlation between the vertical Lorentz force and the respective minimum cross-field current density. Both the error bars of $\left|F_{z}\right|$ (horizontal lines) and of $J_{\perp_{h}}$ (vertical lines) are shown. (c) Correlation between the magnetic field strength, the mean values of the minimum cross-field current density, and the mean vertical current density. The magnetic field values correspond to the minimum values of each sample, while the points and the error bars correspond to the mean $J_{\perp_{h}}$ (rectangles) and $\left|J_{z}\right|$ (triangles) of each sample. The overall mean $\bar{J}_{\perp_{h}}\left(\left|\bar{J}_{z}\right|\right)$ is indicated by a solid (dashed) line, while the shaded area surrounding the line indicates the uncertainty associated with the overall mean. The various samples in $(b)$ and $(c)$ have been selected for $\epsilon\left(F_{z}\right)<0.5$. vertical magnetic field gradient under the assumption of spacefilling magnetic fields and minimum sheath currents flowing on the surface of the emerged flux tubes in low altitudes. No assumption of force-free magnetic fields was employed. Besides being very helpful for the resolution of the $180^{\circ}$ ambiguity, the minimum structure approximation and the corresponding vertical gradient $(\partial B / \partial z)_{p}$ (eq. [6]) reproduce the qualitative characteristics of the variation of the magnetic field with height, as revealed by a rare direct comparison between chromospheric and photospheric magnetic fields (Leka \& Metcalf 2003). Therefore, our predicted $(\partial B / \partial z)_{p}$ should be considered a good approximation of the actual $\partial B / \partial z$. To illustrate the similarities and differences between the predicted gradient $(\partial B / \partial z)_{p}$ and the gradient $(\partial B / \partial z)_{\text {ff }}$, known to hold by identity in the case of force-free fields, substitute $\boldsymbol{B}$ and $B_{z}$ by $B \hat{\boldsymbol{b}}$ and $B b_{z}$, respectively, in equation (3). Simple considerations then give

$$
\left(\frac{\partial B}{\partial z}\right)_{\mathrm{ff}}=b_{h}^{2}\left(\frac{\partial B}{\partial z}\right)_{p}+b_{z}^{2} \frac{\partial B}{\partial z}+B \hat{\boldsymbol{b}} \cdot \nabla b_{z},
$$

where $b_{h}=\left(b_{x}^{2}+b_{y}^{2}\right)^{1 / 2}$ and $\partial B / \partial z$ is the actual vertical field gradient and we have substituted equation (6) for $(\partial B / \partial z)_{p}$. Either assuming $\partial B / \partial z=(\partial B / \partial z)_{\mathrm{ff}}$ in case of force-free fields, or assuming $\partial B / \partial z=(\partial B / \partial z)_{p}$, when applying the minimum structure approximation equation (11) yields

$$
\left(\frac{\partial B}{\partial z}\right)_{\mathrm{ff}}=\left(\frac{\partial B}{\partial z}\right)_{p}+\frac{B}{b_{t}^{2}} \hat{\boldsymbol{b}} \cdot \nabla b_{z},
$$

where $b_{t}=b_{h}$ in the first case and $b_{t}=1$ in the second case. Equation (12) suggests that the minimum structure vertical field gradient is rather incompatible with the force-free approximation, unless $b_{z}=0$. For horizontal fields, $(\partial B / \partial z)_{\mathrm{ff}}=$ $(\partial B / \partial z)_{p}=0$ (eqs. [3] and [6]), so $F_{z}=0$. This, however, does not necessarily mean that the magnetic fields are force-free, since $F_{x}, F_{y}$ may well be nonzero. For the two vertical gradients to match in the general case $b_{z} \neq 0$, one must accept $\hat{\boldsymbol{b}} \cdot \nabla b_{z}=0$. Since $b_{z}=\cos \theta$, where $\theta$ is the zenith angle of the magnetic field vector, the two gradients are identical when $\theta$ remains invariant along the magnetic field lines. This is difficult to visualize for curved magnetic field lines, and especially for closed field lines with both footpoints rooted in the photosphere, unless the radius of curvature of these lines is very large compared to the length of the field lines. While this assumption is not a sufficient condition for $(\partial B / \partial z)_{\mathrm{ff}}=$ $(\partial B / \partial z)_{p}$, in some cases it might allow $\theta$ to vary slowly along the magnetic field lines and the above equation to hold approximately. In Paper I (Appendix B) we have shown that $(\partial B / \partial z)_{\mathrm{ff}}=(\partial B / \partial z)_{p}$, and hence $\hat{\boldsymbol{b}} \cdot \nabla b_{z}=0$, in some special cases of nonradial, axisymmetric, force-free fields such as the Gold-Hoyle and the Lundquist solutions. These idealized cases, however, should be considered the exception rather than the rule, so in general $(\partial B / \partial z)_{\mathrm{ff}} \neq(\partial B / \partial z)_{p}$. Because of this, and because $(\partial B / \partial z)_{p}$ appears to be a reasonable estimate of the actual $\partial B / \partial z$, one might think that a force-free state is hard to attain in the solar atmosphere, especially on or close to the photosphere where the plasma and the magnetic pressures are comparable. In the chromosphere and especially in the corona the insufficient plasma pressure may actually enforce the forcefree condition macroscopically, but small-scale net cross-field currents may still be flowing, particularly in the case of a turbulent evolution within coronal flux tubes (see, e.g., Georgoulis et al. 1998). 
TABLE 1

Synopsis of the Mean Absolute Vertical Lorentz Force, the Mean Value of the Minimum Cross-Field Electric Current Density, and the Mean Absolute Vertical Current Density

\begin{tabular}{|c|c|c|c|c|c|c|}
\hline NOAA AR & Date & Time & $\begin{array}{c}\text { Flux Fraction } \\
\text { for } \epsilon\left(F_{z}\right)<0.5 \\
(\%)\end{array}$ & $\begin{array}{c}\left|\bar{F}_{z}\right| \\
\left(10^{-4} \mathrm{dyn} \mathrm{cm}^{-3}\right)\end{array}$ & $\begin{array}{c}\bar{J}_{\perp_{h}} \\
\left(\mathrm{~mA} \mathrm{~m}^{-2}\right)\end{array}$ & $\begin{array}{c}\left|\bar{J}_{z}\right| \\
\left(\mathrm{mA} \mathrm{m}^{-2}\right)\end{array}$ \\
\hline 10030 & $2002 \mathrm{Jul} 15$ & $19: 50$ & 63 & $2.2 \pm 0.7$ & $44 \pm 20$ & $12 \pm 1.7$ \\
\hline 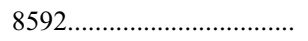 & 1999 Jun 22 & $17: 42$ & 20 & $2.2 \pm 0.6$ & $34 \pm 14$ & $13.8 \pm 1.6$ \\
\hline 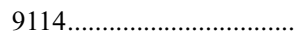 & 2000 Aug 8 & $17: 39-21: 47$ & 99.9 & $0.24 \pm 0.02$ & $12.1 \pm 1.7$ & $3.1 \pm 0.1$ \\
\hline
\end{tabular}

NoтE.-Synopsis of the mean absolute vertical Lorentz force, the mean value of the minimum cross-field electric current density, and the mean absolute vertical current density in our three subject ARs for $\epsilon\left(F_{z}\right)<0.5$. The percentage flux fraction is the fraction of the total well-measured magnetic flux in the AR that is allowed to be used in the calculations based on the above error criterion.

Using the minimum-structure vertical field gradient $(\partial B / \partial z)_{p}$, we calculate the vertical Lorentz force in solar ARs. Typical values range from a few to several times $10^{-4}$ dyn $\mathrm{cm}^{-3}$ and rarely exceed $\sim 10^{-3}$ dyn $\mathrm{cm}^{-3}$. These amplitudes are a few hundredths to a few tenths, at most, of the typical photospheric gravitational force, $F_{g} \sim 9.5 \times 10^{-3} \mathrm{dyn}_{\mathrm{cm}^{-3}}$, calculated for a photospheric mass density $\sim 3.5 \times 10^{-7} \mathrm{~g} \mathrm{~cm}^{-3}$ and a gravitational acceleration $\sim 2.74 \times 10^{4} \mathrm{~cm} \mathrm{~s}^{-2}$. Nevertheless, these Lorentz forces are sufficient to distort significantly the photospheric magnetic field lines given the large scale lengths of the photospheric magnetic fields (Leka \& Metcalf 2003; Paper I).

We have stressed throughout the paper that it is difficult to obtain a reasonably error-free estimate of the vertical Lorentz force, $F_{z}$, and hence of the minimum cross-field current density $J_{\perp_{h}}$, in plages. This is because of the distinct magnetic field geometry in these areas, where the magnetic field vector is almost purely normal to the photosphere (Martinez Pillet et al. 1997). For purely vertical fields, $F_{z}=0$ by definition. Moreover, the relative error, $\epsilon\left(B_{h}\right)$, becomes very large in plages, since $B_{h} \simeq 0$, and precludes a precise calculation of $F_{z}$ and $J_{\perp_{h}}$.

Excluding plages, we have calculated typical cross-field current densities reaching values of several times $10 \mathrm{~mA} \mathrm{~m}^{-2}$, within reasonable uncertainties. Taking these uncertainties into account, cross-field currents are found to be 2-3 times larger than typical vertical current densities, which are on the order of $\sim 15 \mathrm{~mA} \mathrm{~m}^{-2}$. Our estimates of the vertical current density are in line with most previous studies. Therefore, if magnetic flux tubes in the solar atmosphere carry significant currents (Leka et al. 1996) and the minimum possible cross-field currents are a few times larger that the vertical component of the current density, then they should be comparable to the total current density. Hence, cross-field currents are quite significant and the photospheric AR magnetic fields cannot be force-free.

We reach the same conclusion for two morphologically very different ARs: the dynamic, flaring NOAA AR 10030 and the quiescent, nonflaring, NOAA AR 8592. In absolute values of the vertical Lorentz force and the minimum cross-field current density, NOAA AR 10030 is found to be more forced than NOAA AR 8592, as might be expected from the significant complexity and the stronger magnetic fields of the former as compared to the latter AR. The mean vertical current density is very similar in both ARs, on the order of $12-14 \mathrm{~mA} \mathrm{~m}^{-2}$, while $\bar{J}_{\perp_{h}} \simeq 45 \mathrm{~mA} \mathrm{~m}^{-2}$ for NOAA AR 10030 and $\bar{J}_{\perp_{h}} \simeq$ $35 \mathrm{~mA} \mathrm{~m}^{-2}$ for NOAA AR 8592. Hence, both ARs cannot be force-free.

Another issue our conclusions are subject to is the quality of the observations. We have addressed this problem by averaging over several magnetograms of NOAA AR 9114. Averaging significantly improves the signal-to-noise ratio (Fig. 6).
Applying the above analysis to the averaged magnetogram, we reach the same qualitative conclusion as for NOAA ARs 10030 and 8592: the averaged magnetic fields cannot be force-free. In absolute values, the means of the vertical Lorentz force, the minimum cross-field current density, and the vertical current density, together with their uncertainties, are lower in the averaged magnetogram. The calculation with averaged magnetic fields in plages tends to produce reasonable errors in the strong-field parts of these areas, because $\boldsymbol{B}$ is not purely vertical and the uncertainties $\delta B_{h}$ of the horizontal field are substantially decreased by the averaging. As a result, the Lorentz force and the cross-field current density can be estimated in plages for the first time. The vertical Lorentz force in plages is small, as expected from the geometry of the magnetic field vector. Calculating the cross-field current density, however, we obtain similar values as compared to the rest of the AR, because the horizontal magnetic field is weak (eq. [5]).

Despite the lower mean values, the ratio of the mean crossfield current density to the mean vertical current density in the averaged magnetogram of NOAA AR 9114 ranges between 4 and 7, so it is larger than that found for the single magnetograms of NOAA ARs 10030 and 8592. Therefore, the magnitudes of the Lorentz force and the current densities have been decreased in the averaged magnetogram because of the weaker magnetic fields used in the calculations, but the non-force-free character of the AR appears to be enhanced after averaging.

We conclude that, independent of the complexity of the magnetic configuration, the photospheric AR magnetic fields cannot be force-free. It is not possible to determine whether field-aligned currents are stronger than cross-field currents or otherwise, but it appears safe to conclude that a significant part of the free energy in the photospheric part of an AR is carried by cross-field currents. Therefore, at least a part of the energy released in dissipative processes in the low solar atmosphere should stem from the dissipation of cross-field electric currents. Furthermore, if the ideal equilibrium state of minimum magnetic energy in an AR is the linear force-free state (Taylor 1974; however, see Antiochos et al. 2002), then relaxation in solar eruptions should primarily involve dissipation of crossfield currents. We feel that force-free extrapolations of photospheric magnetic fields can still be useful for qualitative purposes and in order to assess, to some extent, the actual three-dimensional magnetic configuration in an AR by means of a relatively simple, inexpensive analytical calculation (see, e.g., the recent results of Pariat et al. 2004). However, the inherent uncertainties and inconsistencies of the force-free approximation, concluded by several authors, will be efficiently dealt with only when the chromospheric magnetic field is used as the boundary of the force-free extrapolations. Until 
chromospheric magnetograms become routinely available, alternative avenues might include more sophisticated non-linear force-free extrapolations (e.g., Regnier et al. 2002), non-forcefree extrapolations based on three-dimensional magnetohydrodynamic simulations (Lionello et al. 2002), or the corrected "quasi-force-free" approach proposed by Berton (2000).
We thank D. M. Rust for a critical reading and several suggestions on the manuscript. Data used here from Mees Solar Observatory, University of Hawaii, are produced with the support of NASA and AFRL grants. This work has received partial support by NSF grant ATM 02-08104, and NASA grants NAG513504 and NAG513021.

\section{APPENDIX}

\section{ERROR PROPAGATION}

The heliographic magnetic field vector $\boldsymbol{B}^{h}$ and the line-of-sight magnetic field vector $\boldsymbol{B}^{i}$ on the image plane (the plane of the observations) can be related through the matrix equation $\boldsymbol{B}^{h}=\mathcal{T} \boldsymbol{B}^{i}$. Here $\mathcal{T}$ is the reference system transformation matrix, given by

$$
\mathcal{T}=\left(\begin{array}{lll}
\alpha_{x 1}, & \alpha_{x 2}, & \alpha_{x 3} \\
\alpha_{y 1}, & \alpha_{y 2}, & \alpha_{y 3} \\
\alpha_{z 1}, & \alpha_{z 2}, & \alpha_{z 3}
\end{array}\right), \text { while } \boldsymbol{B}^{h}=\left(\begin{array}{c}
B_{x} \\
B y \\
B z
\end{array}\right) \text {, and } \boldsymbol{B}^{i}=\left(\begin{array}{c}
B_{\xi} \\
B_{\eta} \\
B_{l}
\end{array}\right)
$$

Each of the matrix terms $\alpha_{m j}(m \equiv\{x, y, z\}, j \equiv\{1,2,3\})$ can be calculated at each location of the image plane by means of the heliographic latitude and longitude of the point, the heliographic latitude and longitude of the center of the solar disk and the angular position of the northern extremity of the solar rotation axis, measured eastward from the northernmost point of the solar disk (Gary \& Hagyard 1990). From equation (A1), the heliographic components $B_{m}(m \equiv\{x, y, z\})$ of the field are given by

$$
B_{m}=\alpha_{m 1} B_{\xi}+\alpha_{m 2} B_{\eta}+\alpha_{m 3} B_{l}
$$

and hence the maximum uncertainty $\delta B_{m}$ of $B_{m}$ is given by

$$
\delta B_{m}=\left|\alpha_{m 1}\right| \delta B_{\xi}+\left|\alpha_{m 2}\right| \delta B_{\eta}+\left|\alpha_{m 3}\right| \delta B_{l}
$$

The uncertainty $\delta B_{l}$ of the longitudinal magnetic field $B_{l}$ is calculated from the fitting of the Stokes profiles. For $\delta B_{\xi}$ and $\delta B_{\eta}$, however, additional considerations are required:

1. From $B_{\xi}=B_{\mathrm{tr}} \cos \left(\phi_{l}\right)$, the maximum uncertainty $\delta B_{\xi}$ is given by

$$
\delta B_{\xi}=\left|\cos \left(\phi_{l}\right)\right| \delta B_{\mathrm{tr}}+B_{\mathrm{tr}}\left|\sin \left(\phi_{l}\right)\right| \delta \phi_{l}
$$

2. From $B_{\eta}=B_{\text {tr }} \sin \left(\phi_{l}\right)$, the maximum uncertainty $\delta B_{\eta}$ is given by

$$
\delta B_{\eta}=\left|\sin \left(\phi_{l}\right)\right| \delta B_{\mathrm{tr}}+B_{\mathrm{tr}}\left|\cos \left(\phi_{l}\right)\right| \delta \phi_{l}
$$

where both $\delta B_{\text {tr }}$ and $\delta \phi_{l}$ are obtained from the fitting of the Stokes profiles.

From equation (A3) and for $\delta B_{\xi}, \delta B_{\eta}$ given by equations (A4) and (A5), respectively, one finds the uncertainties $\delta B_{m}$ ( $m \equiv\{x, y, z\})$ of the heliographic magnetic field components. The uncertainties $\delta B_{z}$ in the vertical magnetic field are given by

$$
\delta B_{z}=\left|\alpha_{z 1}\right| \delta B_{\xi}+\left|\alpha_{z 2}\right| \delta B_{\eta}+\left|\alpha_{z 3}\right| \delta B_{l} .
$$

On or close to disk center, equation (A6) implies that $\delta B_{z} \simeq \delta B_{l}$, as a result of $B_{z} \simeq B_{l}$ in this case. More noisy vertical fields are obtained far from disk center, since $\delta B_{l}$ is typically smaller than $\delta B_{\text {tr }}$ and the latter increasingly influences the uncertainty $\delta B_{z}$ as the

location of consideration moves away from disk center.
Let us now focus on the horizontal magnetic field, $B_{h}=\left(B_{x}^{2}+B_{y}^{2}\right)^{1 / 2}$. From the matrix transformation to the heliographic reference system and equation (A2), we find

$$
\begin{aligned}
B_{h}= & {\left[\left(\alpha_{x 1}^{2}+\alpha_{y 1}^{2}\right) B_{\xi}^{2}+\left(\alpha_{x 2}^{2}+\alpha_{y 2}^{2}\right) B_{\eta}^{2}+\left(\alpha_{x 3}^{2}+\alpha_{y 3}^{2}\right) B_{l}^{2}+2\left(\alpha_{x 1} \alpha_{x 3}+\alpha_{y 1} \alpha_{y 3}\right) B_{\xi} B_{l}\right.} \\
& \left.+2\left(\alpha_{x 1} \alpha_{x 2}+\alpha_{y 1} \alpha_{y 2}\right) B_{\xi} B_{\eta}+2\left(\alpha_{x 2} \alpha_{x 3}+\alpha_{y 2} \alpha_{y 3}\right) B_{\eta} B_{l}\right]^{1 / 2}
\end{aligned}
$$


An upper limit, $\delta B_{h}$, of the uncertainties of $B_{h}$ is then given by

$$
\delta B_{h}=\left|\frac{\partial B_{h}}{\partial B_{\xi}}\right| \delta B_{\xi}+\left|\frac{\partial B_{h}}{\partial B_{\eta}}\right| \delta B_{\eta}+\left|\frac{\partial B_{h}}{\partial B_{l}}\right| \delta B_{l},
$$

where

$$
\begin{aligned}
& \frac{\partial B_{h}}{\partial B_{\xi}}=\left(\frac{1}{B_{h}}\right)\left[\left(\alpha_{x 1}^{2}+\alpha_{y 1}^{2}\right) B_{\xi}+\left(\alpha_{x 1} \alpha_{x 3}+\alpha_{y 1} \alpha_{y 3}\right) B_{l}+\left(\alpha_{x 1} \alpha_{x 2}+\alpha_{y 1} \alpha_{y 2}\right) B_{\eta}\right], \\
& \frac{\partial B_{h}}{\partial B_{\eta}}=\left(\frac{1}{B_{h}}\right)\left[\left(\alpha_{x 2}^{2}+\alpha_{y 2}^{2}\right) B_{\eta}+\left(\alpha_{x 1} \alpha_{x 2}+\alpha_{y 1} \alpha_{y 2}\right) B_{\xi}+\left(\alpha_{x 2} \alpha_{x 3}+\alpha_{y 2} \alpha_{y 3}\right) B_{l}\right], \\
& \frac{\partial B_{h}}{\partial B_{l}}=\left(\frac{1}{B_{h}}\right)\left[\left(\alpha_{x 3}^{2}+\alpha_{y 3}^{2}\right) B_{l}+\left(\alpha_{x 1} \alpha_{x 3}+\alpha_{y 1} \alpha_{y 3}\right) B_{\xi}+\left(\alpha_{x 2} \alpha_{x 3}+\alpha_{y 2} \alpha_{y 3}\right) B_{\eta}\right],
\end{aligned}
$$

and $\delta B_{\xi}$ and $\delta B_{\eta}$ are given by equations (A4) and (A5), respectively. Close to disk center, $\delta B_{h} \simeq \delta B_{\text {tr }}$, since $B_{h} \simeq B_{\text {tr }}$ in this case.

For simplicity in the dimensional calculations that follow, we assume $\delta B_{x} \simeq \delta B_{y} \simeq \delta B_{h}$. This assumption both simplifies the calculations and yields an upper limit of the uncertainties, since $\delta B_{h} \gtrsim \delta B_{x}$ and $\delta B_{h} \gtrsim \delta B_{y}$ at every location of a given vector magnetogram, as $B_{h}$ comprises both $B_{x}$ and $B_{y}$.

Knowledge of $\delta B_{z}$ and $\delta B_{h}$ at every point of a vector magnetogram allows the calculation of the errors in the minimum structure vertical field gradient, the vertical Lorentz force, the minimum cross-field current density, and the vertical current density as follows:

1. For the minimum-structure vertical field gradient $(\partial B / \partial z)_{p}$, equation (6) implies that

$$
\left(\frac{\partial B}{\partial z}\right)_{p}=\frac{B_{z}}{B_{h}^{2}}\left(B_{x} \frac{\partial B}{\partial x}+B_{y} \frac{\partial B}{\partial y}\right)
$$

Letting $\partial B / \partial x \simeq \partial B / \partial y \sim B / \lambda$, where $\lambda$ is the size of the magnetogram's pixel and $B_{x} \simeq B_{y} \sim B_{h}$, equation (A10) implies that

$$
\left|\left(\frac{\partial B}{\partial z}\right)_{p}\right| \approx 2 \frac{B_{z} B}{\lambda B_{h}}
$$

It is then trivial to show that the relative error $\epsilon(\partial B / \partial z)=\left[\delta(\partial B / \partial z) /|\partial B / \partial z|_{p}\right]$ of the vertical field gradient, $(\partial B / \partial z)_{p}$, is given by

$$
\epsilon\left(\frac{\partial B}{\partial z}\right)=\epsilon\left(B_{z}\right)+\epsilon\left(B_{h}\right)+\epsilon(B)
$$

where $\epsilon\left(B_{z}\right)=\delta B_{z} /\left|B_{z}\right|, \epsilon\left(B_{h}\right)=\delta B_{h} / B_{h}$, and $\epsilon(B)=\delta B / B$ are the relative errors of $B_{z}, B_{h}$, and $B$, respectively. The uncertainties, $\delta B$, of the magnetic field strength, $B=\left(B_{l}^{2}+B_{\mathrm{tr}}^{2}\right)^{1 / 2}$, on the other hand, are given by

$$
\delta B=\frac{\left|B_{l}\right|}{B} \delta B_{l}+\frac{B_{\mathrm{tr}}}{B} \delta B_{\mathrm{tr}}
$$

2. For the vertical Lorentz force $F_{z}$, equation (4) implies that

$$
F_{z}=\frac{1}{4 \pi}\left[B_{x} \frac{\partial B_{z}}{\partial x}+B_{y} \frac{\partial B_{z}}{\partial y}-B_{z}\left(\frac{\partial B_{x}}{\partial x}+\frac{\partial B_{y}}{\partial y}\right)-B \frac{\partial B}{\partial z}\right]
$$

Letting $\partial B_{m} / \partial n \sim B_{m} / \lambda(m, n \equiv\{x, y, z\})$ and $B_{x} \simeq B_{y} \sim B_{h}$, equation (A14) gives

$$
\left|F_{z}\right| \approx \frac{1}{\pi \lambda}\left|B_{z}\right| B_{h}+\frac{1}{4 \pi} B\left|\left(\frac{\partial B}{\partial z}\right)_{p}\right| .
$$

The relative error $\epsilon\left(F_{z}\right)=\delta F_{z} /\left|F_{z}\right|$ can then be obtained, where an upper limit $\delta F_{z}$ of the uncertainties in the vertical Lorentz force is given by

$$
\delta F_{z}=\frac{1}{\pi \lambda}\left|B_{z}\right| \delta B_{h}+\frac{1}{\pi \lambda} B_{h} \delta B_{z}+\frac{1}{4 \pi}\left|\left(\frac{\partial B}{\partial z}\right)_{p}\right| \delta B+\frac{1}{4 \pi} B \delta\left(\frac{\partial B}{\partial z}\right) .
$$


The minimum-structure vertical field gradient in equation (A16) is given by equation (A11).

3. For the minimum cross-field current density $J_{\perp_{h}}$, equation (5) directly implies a relative error, $\epsilon\left(J_{\perp_{h}}\right)$, given by

$$
\epsilon\left(J_{\perp_{h}}\right)=\epsilon\left(F_{z}\right)+\epsilon\left(B_{h}\right) .
$$

4. Applying Ampère's law for the vertical current density, $J_{z}$, we find

$$
J_{z}=\frac{c}{4 \pi}\left(\frac{\partial B_{y}}{\partial x}-\frac{\partial B_{x}}{\partial y}\right)
$$

Letting $\partial B_{m} / \partial n \sim B_{m} / \lambda(m, n \equiv\{x, y\})$ and $B_{x} \simeq B_{y} \sim B_{h}$, equation (A18) implies that

$$
\left|J_{z}\right| \approx \frac{c B_{h}}{2 \pi \lambda} .
$$

From equation (A19), it then follows that the relative error $\epsilon\left(J_{z}\right)$ is thus

$$
\epsilon\left(J_{z}\right)=\epsilon\left(B_{h}\right) .
$$

Alissandrakis, C. E. 1981, A\&A, 100, 197

Aly, J. J. 1984, ApJ, 283, 349

Amari, T., Boulmezaoud, T. Z., \& Mikić, Z. 1999, A\&A, 350, 1051

Antiochos, S. K., Carpen, J. T., \& DeVore, C. R. 2002, ApJ, 575, 578

Berton, R. 2000, A\&A, 356, 301

Brosius, J. W., Davila, J. M., Thomas, R. J., \& White, S. M. 1997, ApJ, 488, 488

Cuperman, S., Bruma, C., \& Heristchi, D. 1997, A\&AS, 122, 381

Cuperman, S., Li, J., \& Semel, M. 1992, A\&A, 265, 296 1993, A\&A, 268, 749

de La Beaujardière, J.-F., Canfield, R. C., \& Leka, K. D. 1993, ApJ, 411, 378 Ding, Y. J., Hong, Q. F., Hagyard, M. J., Deloach, A. C., \& Liu, X. P. 1987, Sol. Phys., 109, 307

Gary, G. A. 1989, ApJS, 69, 323

Gary, G. A., \& Démoulin, P. 1995, ApJ, 445, 982

Gary, G. A., \& Hagyard, M. J. 1990, Sol. Phys., 126, 21

Gary, G. A., \& Moore, R. L. 2004, ApJ, 611, 545

Georgoulis, M. K., LaBonte, B. J., \& Metcalf, Th. R. 2004, ApJ, 602, 446 (Paper I)

Georgoulis, M. K., Velli, M., \& Einaudi, G. 1998, ApJ, 497, 957

Jackson, J. D. 1962, Classical Electrodynamics (New York: Wiley)

Krall, K. R., Smith, J. B., Jr., Hagyard, M. J., West, E. A., \& Cummings, N. P. 1982, Sol. Phys., 79, 59

LaBonte, B. J. 2002, in Proc. Yohkoh 10th Anniversary Meeting, Multiwavelength Observations of Coronal Structure and Dynamics, ed. P. C. H. Martens \& D. P. Cauffman (Amsterdam: Pergamon), 87

Leka, K. D., Canfield, R. C., McClymont, A. N., \& van Driel-Gesztelyi, L. 1996, ApJ, 462, 547

\section{REFERENCES}

Leka, K. D., \& Metcalf, Th. R. 2003, Sol. Phys., 212, 361

Leka, K. D., \& Skumanich, A. 1999, Sol. Phys., 188, 3

Li, J., Metcalf, Th. R., Canfield, R. C., \& Wülser, J.-P. 1997, ApJ, 482, 490

Lin, H., Penn, M. J., \& Kuhn, J. R. 1998, ApJ, 493, 978

Lin, H., Penn, M. J., \& Tomczyk, S. 2000, ApJ, 541, L83

Lionello, R., Mikić, Z., Linker, J. A., \& Amari, T. 2002, ApJ, 581, 718

Liu, Y., Jiang, Y., Ji, H., Zhang, H., \& Wang, H. 2003, ApJ, 593, L137

Low, B. C. 1982, Rev. Geophys. Space Phys., 20, 145

Low, B. C., \& Lou, Y. Q. 1990, ApJ, 352, 343

Martinez Pillet, V., Lites, B. W., \& Skumanich, A. 1997, ApJ, 474, 810

McClymont, A. N., Jiao, L., \& Mikić, Z. 1997, Sol. Phys., 174, 191

Metcalf, Th. R., Canfield, R. C., Hudson, H. S., Mickey, D. L., Wülser, J.-P., Martens, P. C. H., \& Tsuneta, S. 1994, ApJ, 428, 860

Metcalf, Th. R., Jiao, L., McClymont, A. N., Canfield, R. C., \& Uitenbroek, H. 1995, ApJ, 439, 474

Mickey, D. L., Canfield, R. C., LaBonte, B. J., Leka, K. D., Waterson, M. F., \& Weber, H. M. 1996, Sol. Phys., 168, 229

Moon, Y.-J., Choe, G. S., Yun, H. S., Park, Y. D., \& Mickey, D. L. 2002, ApJ, 568,422

Pariat, E., Aulanier, G., Schmieder, B., Georgoulis, M. K., Rust, D. M., \& Bernasconi, P. N. 2004, ApJ, in press

Pizzo, V. J. 1986, ApJ, 302, 785

Regnier, S., Amari, T., \& Kersalé, E. 2002, A\&A, 392, 1119

Taylor, J. B. 1974, Phys. Rev. Lett., 33, 1139

Zhang, H. 2001, ApJ, 557, L71 